\title{
Aceleraciones de piso para diseño de elementos no estructurales y estructurales que no hacen parte del sistema de resistencia sísmica en edificios*
}

\author{
Ricardo E. Barbosa ${ }^{* *}$ \\ José J. Álvarez E. ${ }^{* * *}$ \\ Julián Carrillo ${ }^{* * * *}$
}

\author{
Recibido: 05/10/2017 • Aceptado: 25/06/2018 \\ https://doi.org/10.22395/rium.v17n33a5
}

\begin{abstract}
Resumen
El propósito del artículo es evaluar el método usado en el Reglamento colombiano de construcción sismo resistente (NSR-10) para calcular las aceleraciones de piso que son necesarias para diseñar elementos no estructurales y elementos estructurales que no hacen parte del sistema de resistencia sísmica. En el estudio se comparan las aceleraciones máximas de pisos calculadas con las normas NSR-10, ASCE 7-10, UBC-97, el Eurocódigo 8-04 y NZS 1170.5-04, con las aceleraciones máximas de piso medidas en especímenes ensayados en mesa vibratoria, y en edificaciones existentes durante sismos reales. En el artículo también se propone una modificación al método actualmente utilizado por la NSR-10. La modificación propuesta genera una estimación más acertada de las aceleraciones necesarias para diseñar estos elementos en edificios de mediana y gran altura. Las recomendaciones propuestas se fundamentan en los resultados de la evaluación del método usado actualmente en la NSR-10, el procedimiento usado en otras normas sismorresistentes, en resultados experimentales medidos en modelos de estructuras de concreto reforzado y en aceleraciones registradas en edificios instrumentados.
\end{abstract}

Palabras clave: aceleración de piso; contenido de edificios; daño por sismo; edificio instrumentado; elemento no estructural; sistema de resistencia sísmica.

Artículo de investigación terminada. Proyecto financiado por UMNG y EngSolutions Inc., vigencia 2016-2017.

** Ph. D. Profesor de cátedra (Adjunct Professor) en Florida Atlantic University, School of Architecture. Presidente de EngSolutions, Inc. 8170 SW 29th Ct, Ft Lauderdale, FL 33328, USA. Correo electrónico: barbosar@fau.edu. Orcid: https://ORCID.org/0000-0002-0217-3664

*** Magíster en Ingeniería Civil. Presidente de la Comisión técnica de estructuras y construcción de edificios, Sociedad Colombiana de Ingenieros, SCI. Carrera 14b N. ${ }^{\circ}$ 112-60, Bogotá, Colombia. Correo electrónico: jjalvarezenciso@gmail.com. Orcid: https://orcid.org/0000-0001-8957-8463

**** Doctor en Ingeniería. Profesor titular de la Universidad Militar Nueva Granada, UMNG. Grupo de investigación Estructuras y Sísmica. Carrera 11, No. 101-80. Edificio F, Piso 2. Correo electrónico: wjcarrillo@gmail.com. Orcid: https://orcid.org/0000-0002-8274-5414 


\title{
Floor Accelerations for the Design of Non-Structural and Structural Elements that are not Part of the Seismic Resistance System in Buildings
}

\begin{abstract}
The purpose is to evaluate the method used in the Colombian Earthquake Resistant Construction Regulations (NSR-10) to calculate the floor accelerations that are necessary to design non-structural elements and structural elements that are not part of the seismic resistance system. The study compares the maximum floor accelerations calculated with NSR-10, ASCE 7-10, UBC-97, Eurocode 8-04 and NZS 1170.5-04, with the maximum floor accelerations measured in specimens tested on a vibrating table, and in existing buildings during real earthquakes. The article also proposes a modification to the method currently used by NSR-10. The proposed modification generates a more accurate estimate of the accelerations needed to design these elements in medium and high-rise buildings. The proposed recommendations are based on the results of the evaluation of the method currently used in NSR-10, the procedure used in other seismic-resistant standards, experimental results measured in reinforced concrete structure models and accelerations recorded in instrumented buildings.
\end{abstract}

Keywords: floor acceleration; building contents; earthquake damage; instrumented building; non-structural element; seismic resistance system.

\section{Acelerações de piso para o desenho de elementos não estruturais e estruturais que não fazem parte do sistema de resistência sísmica em edifícios}

\begin{abstract}
Resumo
O propósito do artigo é avaliar o método usado no Regulamento colombiano de construção sismorresistente (NSR-10) para calcular as acelerações de piso que são necessárias para desenhar elementos não estruturais e elementos estruturais que não fazem parte do sistema de resistência sísmica. No estudo, as acelerações máximas de pisos calculadas com as normas NSR-10, ASCE 7-10, UBC-97, o Eurocódigo 8-04 e NZS 1170.5-04 são comparadas com as acelerações máximas de piso medidas em modelos ensaiados em mesa vibratória e em construções existentes durante sismos reais. No artigo também se propõe uma modificação ao método utilizado atualmente pela NSR-10. A modificação proposta gera uma estimativa mais acertada das acelerações necessárias para desenhar esses elementos em edifícios de altura média e grande. As recomendações propostas se fundamentam nos resultados da avaliação do método usado atualmente na NSR-10, no procedimento usado em outras normas sismorresistentes, em resultados experimentais medidos em modelos de estruturas de concreto reforçado e em acelerações registradas em edifícios instrumentados.
\end{abstract}

Palavras-chave: aceleração de piso; conteúdo de edifícios; dano por sismo; edifício instrumentado; elemento não estrutural; sistema de resistência sísmica. 


\section{INTRODUCCIÓN}

La mayor parte del daño causado a edificios por sismos, corresponde a fallas de elementos no estructurales, los cuales incluyen componentes arquitectónicos, mecánicos, eléctricos, hidráulicos y sanitarios, así como también muebles, equipos y contenido de edificios. Algunos ejemplos de componentes no estructurales que son vulnerables a daño durante sismos, cuando no están debidamente anclados, incluyen particiones, fachadas, techos falsos, pisos falsos, artefactos de iluminación, ductos, equipos de aire acondicionado, elevadores, centros de cómputo, equipo de hospital, estanterías y mercancía en general. Para diseño de componentes no estructurales y su protección sísmica, es necesario estimar las aceleraciones de piso, la cuales permiten determinar los efectos inerciales del sismo que causan deslizamiento, volteo y caída de estos elementos.

Aunque varios estudios han propuesto nuevos métodos o modificaciones a las provisiones de los reglamentos para calcular las aceleraciones máximas de piso [1-3], la mayoría de estos están basados en consideraciones teóricas, analíticas o computacionales, y no en evidencia experimental o en mediciones durante sismos reales.

El objetivo de este artículo es evaluar y discutir el método usado en el Reglamento colombiano de construcción sismo resistente NSR-10 [4] para calcular las aceleraciones de piso para diseño de elementos no estructurales y de elementos estructurales que no hacen parte del sistema de resistencia sísmica. Inicialmente, se discuten los daños observados en elementos no estructurales y de elementos no estructurales que no hacen parte del sistema de resistencia sísmica durante los sismos de México (2017), Ecuador (2016), Bucaramanga (2015), Chile (2010), Nueva Zelanda (2011), Quindío (1999) y Northridge (1994). Luego se describen las aceleraciones máximas de pisos calculadas según las normas NSR-10 [4], ASCE 7-10 [5], UBC-97 [6], el Eurocódigo 8-04 [7] y la norma Neozelandesa NZS 1170.5-04 [8]. Estas aceleraciones calculadas se comparan con las aceleraciones máximas de piso medidas experimentalmente en pruebas en mesa vibratoria, y en edificaciones existentes durante sismos reales.

En particular, se presentan y discuten los resultados de cuatro casos de estudio donde se evalúan las aceleraciones de pisos para diseño de componentes no estructurales. En el primer caso se comparan los resultados de las ecuaciones de los reglamentos analizados para predecir las aceleraciones máximas de pisos para un edificio de 32 pisos. En el segundo caso de estudio se comparan las predicciones de los reglamentos con las aceleraciones medidas en ensayos en mesa vibratoria de estructuras a escala reducida con diferentes sistemas estructurales. En el tercer caso de estudio se comparan las predicciones de los reglamentos con las aceleraciones medidas en siete edificaciones de concreto reforzado localizadas en el área de Los Ángeles, California. En el último caso se comparan las predicciones de los reglamentos con las aceleraciones medidas 
en una edificación real de 20 pisos localizado en el norte de Hollywood, California. Finalmente, en el artículo se proponen modificaciones puntuales a la NSR-10.

\section{DAÑOS OBSERVADOS EN ELEMENTOS NO ESTRUCTURALES}

El costo de los componentes no estructurales de un edificio comercial (mecánicos, eléctricos, hidráulicos, sanitarios y arquitectónicos) representa una fracción importante de la inversión total de capital en la edificación. Por otra parte, el contenido perteneciente a los ocupantes del edificio, que incluye muebles, equipo médico y de oficina, representa un valor adicional significativo. La falla y caída de estas componentes no solo puede ser costosa sino también peligrosa. El principal peligro asociado a la falla o caída de elementos no estructurales es que puede causar heridos y muertos. El desprendimiento y caída de fachadas, parapetos y particiones de mampostería durante sismos han resultado en la muerte, no solo de ocupantes, sino también de transeúntes. Igualmente, la caída de fachadas en vidrio puede ser peligrosa, especialmente cuando hay áreas acristaladas directamente encima de las salidas (ver fig. 1).
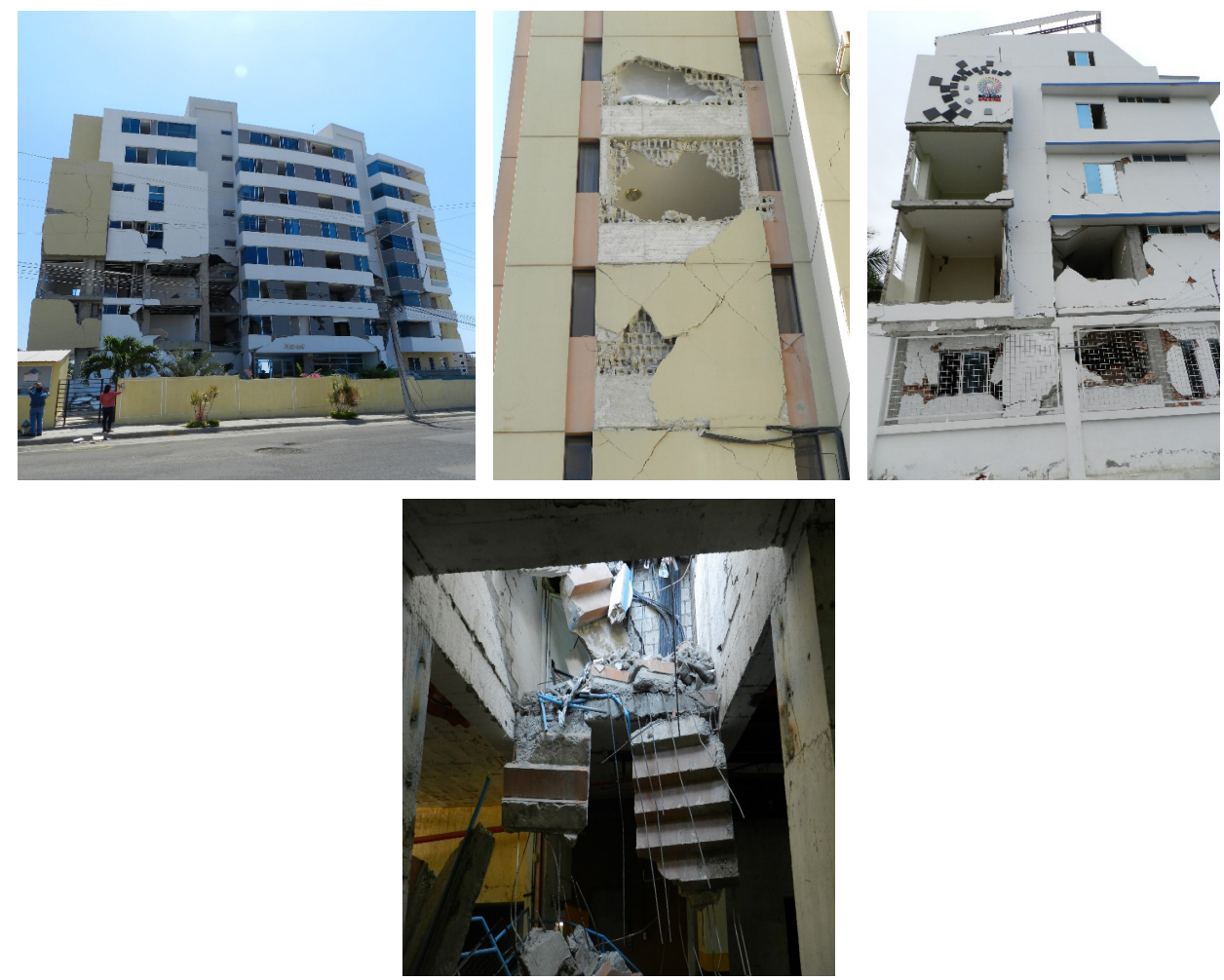

Figura 1. Caída de fachadas y escaleras en el sismo de Ecuador de 2016 Fuente: elaboración propia 
Los daños potencialmente peligrosos de elementos no estructurales incluyen no solo caída de ventiladores y artefactos de iluminación, equipo pesado de ventilación y chimeneas, sino también volteo de gabinetes y bibliotecas altas y pesadas. También se compromete la seguridad cuando los componentes no estructurales bloquean escaleras y salidas de edificios. Además de las significativas pérdidas directas correspondientes al daño de elementos no estructurales que no están anclados apropiadamente para resistir las fuerzas inerciales generadas durante el sismo, la caída de algunos de estos elementos puede resultar en importantes pérdidas indirectas de propiedad (ver fig. 1).

Las fugas de agua de calentadores de agua y de unidades de aire acondicionado que han sido volteadas por el sismo, pueden resultar en importantes daños por agua, los cuales podrían forzar el cierre temporal de edificaciones indispensables. Por ejemplo, el hospital Talca en el sismo de Chile de 2010 tuvo que ser cerrado por los daños causados por el agua. Similarmente, la combinación de fallas en componentes eléctricos y de fugas de gas causadas por calentadores de agua anclados inadecuadamente, que al voltearse producen la rotura de la tubería de suministro de gas, frecuentemente originan incendios que también resultan en costosos daños.

Los soportes de la red de rociadores contra incendio deben ser diseñados para que resistan los efectos inerciales del sismo. El diseño de estos elementos es importante porque la pérdida de protección pone en riesgo la vida de los ocupantes del edificio. Adicionalmente, en varios sismos se ha observado que las fallas en la red de rociadores generan liberación de grandes volúmenes de agua, lo que produce el colapso de cielos rasos y pérdidas importantes de propiedad, todo ello, finalmente, resulta en uno de los tipos de daño no estructural más costosos.

Similarmente, los daños a equipos de cómputo, equipo eléctrico y de comunicaciones por falla, caída o volcamiento o por colapso de pisos falsos (pedestales fallados o volteados), pueden forzar el cierre de instalaciones médicas, centros de atención a la comunidad, negocios y oficinas, mientras se completan reparaciones adecuadas. Por otra parte, el colapso de elementos estructurales que no hace parte del sistema de resistencia sísmica, tales como escaleras y rampas, compromete de varias maneras la seguridad de los ocupantes.

\section{PROVISIONES DE REGLAMENTOS PARA DISEÑO SÍSMICO}

Para diseñar apropiadamente los componentes no estructurales y su protección sísmica (anclaje y arrostramiento) es necesario estimar las aceleraciones de piso. Estas aceleraciones permiten determinar los efectos inerciales del sismo que causan deslizamiento, volteo y caída de estos elementos. Las normas de diseño sismorresistente requieren que las fuerzas sísmicas de diseño $\left(F_{p}\right)$ que actúan sobre cualquier elemento no estructural, se calculen a partir de la ecuación (1). 


$$
F_{p}=\frac{a_{x} a_{p}}{R_{p}} W_{p}
$$

Donde $a_{x}$ es la aceleración en el punto de soporte del elemento expresada como una fracción de la gravedad, $W_{p}$ es el peso del elemento, $a_{p}$ es un factor de amplificación de la aceleración en el elemento y $R_{p}$ es un factor de modificación de respuesta. Las normas especifican un valor mínimo de la fuerza sísmica. La gran diferencia entre la norma NSR-10 [4] y otras normas sísmicas internacionales está en la evaluación de las aceleraciones $a_{x}$. Para elementos estructurales que no hacen parte del sistema de resistencia sísmica se aplica una ecuación similar a la ecuación (1). A continuación, se describe el cálculo de las aceleraciones $a_{x}$ en algunas normas internacionales.

\subsection{Aceleraciones según la NSR-10}

En la sección A.9.4.2.1 de la NSR-10 [4] se define la aceleración en el punto de soporte y se calcula por medio de la ecuación (2).

$$
\begin{array}{rlrl}
a_{x} & =A_{s}+\frac{\left(S_{a}-A_{s}\right) h_{x}}{h_{e q}} & & h_{x}<h_{e q} \\
a_{x}=S_{a} \frac{h_{x}}{h_{e q}} & h_{x}>h_{e q}
\end{array}
$$

Donde $A_{s}$ es la aceleración máxima en la base, $S_{a}$ es la aceleración espectral y $h_{x}$ es la altura del nivel de apoyo del elemento. La altura equivalente $h_{e q}$ puede estimarse a partir de la altura total $\left(h_{n}\right)$ como $0,75 \times h_{n}$. Según la ecuación 2, las aceleraciones de piso varían linealmente desde el valor en la base $A_{s}$ hasta la aceleración espectral $S_{a}$ a la altura equivalente. Desde la altura equivalente hasta el techo, las aceleraciones aumentan con la altura en proporción a la relación de alturas $h_{x} / h_{e q}$.

\subsection{Aceleraciones según la ASCE 7-10}

Según la sección 13.3.1 de la ASCE 7-10 [5], la aceleración en el punto de soporte puede calcularse por medio de la ecuación (3). Esta ecuación fue originada en las Provisiones NEHRP [10-11].

$$
a_{x}=A_{s}\left(1+2 \frac{h_{x}}{h_{n}}\right)
$$

Donde $A_{s}$ es aceleración máxima en la base, $h_{n}$ es la altura del techo y $h_{x}$ es la altura del nivel de apoyo. En ASCE 7-10, el término $A_{s}$ es remplazado por $0.4 \times S_{D S}$. Según la ecuación (3), las aceleraciones de piso siempre aumentan linealmente con la altura y la aceleración en el techo es tres veces mayor que la aceleración en la base. 


\subsection{Aceleraciones según el UBC-97}

En el reglamento UBC-97 [6], el cual estuvo vigente en California hasta el año 2001 y que es la base de varios códigos internacionales, la aceleración en el punto de soporte puede calcularse por medio de la ecuación (4).

$$
a_{x}=A_{s}\left(1+3 \frac{h_{x}}{h_{n}}\right)
$$

Donde $A_{s}$ es la aceleración máxima en la base, $h_{n}$ es la altura del techo y $h_{x}$ es la altura del nivel de apoyo. Según la ecuación (4), las aceleraciones de piso aumentan con la altura y la aceleración en el techo es cuatro veces mayor que la aceleración en la base.

\subsection{Aceleraciones según el Eurocódigo 8-04}

Según el Eurocódigo 8-04 [7], la aceleración en el punto de soporte puede expresarse por medio de la ecuación (5).

$$
a_{x}=A_{s}\left[\frac{3}{2}\left(1+\frac{h_{x}}{h_{n}}\right)-0.5\right]
$$

La ecuación (5) aplica a un elemento no estructural rígido, es decir, no incluye el efecto de amplificación de la componente. En la NSR-10 [4] y ASCE 7-10 [5], esta amplificación está dada por el factor $a_{p}$, mientras que en el Eurocódigo 8-04 [7], la amplificación está representada por el factor del primer término de la ecuación (5). Este término es función de la relación entre el periodo fundamental del elemento $T_{a} \mathrm{y}$ el periodo fundamental del edificio $T_{1}$. La ecuación (5) en este artículo está indicada para $\left(T_{a} / T_{1}\right)=0$. Según esta ecuación, las aceleraciones de piso aumentan con la altura y la aceleración en el techo es 2,5 veces mayor que la aceleración en la base.

\subsection{Aceleraciones según la NZS 1170.5-04}

Según la norma Neozelandesa NZS 1170.5-04 [8], la aceleración en el punto de soporte puede calcularse por medio de la ecuación (6).

$$
\begin{array}{ll}
a_{x}=A_{s}\left(1+2 \frac{h_{x}}{h_{l}}\right) & h_{x}<h_{l} \\
a_{x}=3 A_{s} & h_{x}>h_{l}
\end{array}
$$

Donde la altura límite $\left(h_{l}\right)$ se calcula como el mayor valor entre $12 \mathrm{~m}$ y $0.2 h_{n}$, donde $h_{n}$ es la altura total. Según la ecuación (6), las aceleraciones de piso aumentan 
linealmente con la altura y la aceleración en la altura $h_{l}$ es hasta tres veces el valor de la aceleración en la base. A partir de la altura $h_{l}$ hasta el techo, las aceleraciones máximas son iguales a tres veces la aceleración en la base.

Varios estudios han propuesto nuevos métodos o modificaciones a las provisiones de los reglamentos para calcular las aceleraciones máximas de piso [1-3]. Sin embargo, la mayoría de estos estudios están basados en consideraciones teóricas, analíticas o computacionales y no en evidencia experimental o en mediciones durante sismos reales.

\section{METODOLOGÍA}

La metodología del estudio consiste en comparar las aceleraciones máximas de pisos calculadas según las normas NSR-10 4], ASCE 7-10 [5], UBC-97 [6], el Eurocódigo 8-04 [7] y la norma Neozelandesa NZS 1170.5-04 [8], con las aceleraciones máximas de piso medidas experimentalmente en pruebas en mesa vibratoria y en edificaciones existentes durante sismos reales. En particular, se presentan y discuten los resultados de cuatro casos de estudio donde se evalúan las aceleraciones de pisos para diseño de componentes no estructurales. En el primer caso se comparan los resultados de las ecuaciones de los reglamentos analizados para predecir las aceleraciones máximas de pisos para un edificio de 32 pisos. En el segundo caso de estudio se comparan las predicciones de los reglamentos con las aceleraciones medidas en ensayos en mesa vibratoria de estructuras a escala reducida con diferentes sistemas estructurales. En el tercer caso de estudio se comparan las predicciones de los reglamentos con las aceleraciones medidas en siete edificaciones de concreto reforzado localizadas en el área de Los Ángeles, California. En el último caso se comparan las predicciones de los reglamentos con las aceleraciones medidas en una edificación real de 20 pisos localizado en el norte de Hollywood, California.

\section{RESULTADOS Y DISCUSIÓN}

Para facilitar el análisis de la información, en esta sección se presenta la descripción detallada de los casos de estudio, junto con los resultados y discusión de los resultados obtenidos.

\subsection{Caso de estudio 1: comparación entre predicciones de reglamentos para un edificio de 32 pisos}

Para comparar las aceleraciones de piso que se utilizan en la norma NSR-10 [4] con las aceleraciones propuestas en otras normas [5-8] para diseño de elementos no estructurales, en el primer caso de estudio se analizan los resultados de una estructura 
real de 32 pisos, correspondientes a 28 pisos de apartamentos más cuatro pisos de parqueaderos (aéreos). La estructura está localizada en una zona de amenaza sísmica intermedia. El sistema estructural que resiste las cargas gravitacionales y las fuerzas sísmicas consiste en pórticos de concreto reforzado con capacidad moderada de disipación de energía (DMO). El tamaño de las columnas varía e incluye secciones de $0,60 \times 1,60 \mathrm{~m}, 0,60 \times 2,00 \mathrm{~m}, 0,60 \times 2,20 \mathrm{~m}, 0,70 \times 1,80 \mathrm{~m}$ y $0,80 \times 1,40 \mathrm{~m}$. El tamaño de las vigas varía e incluye secciones de $0,50 \times 0,70 \mathrm{~m}, 0,60 \times 0,60 \mathrm{~m}$ y $0,60 \times 0,70 \mathrm{~m}$. La estructura esta desplantada en un perfil de suelo tipo D. La cimentación consiste en pilas preexcavadas de $25 \mathrm{~m}$ de longitud, las cuales están dispuestas en grupos de 2 y 3 pilas por columna. El modelo matemático del sistema de resistencia sísmica se presenta en la figura 2, junto con un resumen de los parámetros sísmicos y de los resultados del análisis sísmico obtenido con la herramienta computacional RCB V8.5 [12]. Aunque el diseño original de la estructura se basó en un análisis dinámico modal espectral, para un seguimiento más sencillo, en este artículo se presentan resultados de un análisis usando el método de la fuerza horizontal equivalente. Sin embargo, para el caso de la comparación de las aceleraciones, las conclusiones son las mismas con los dos métodos de análisis.
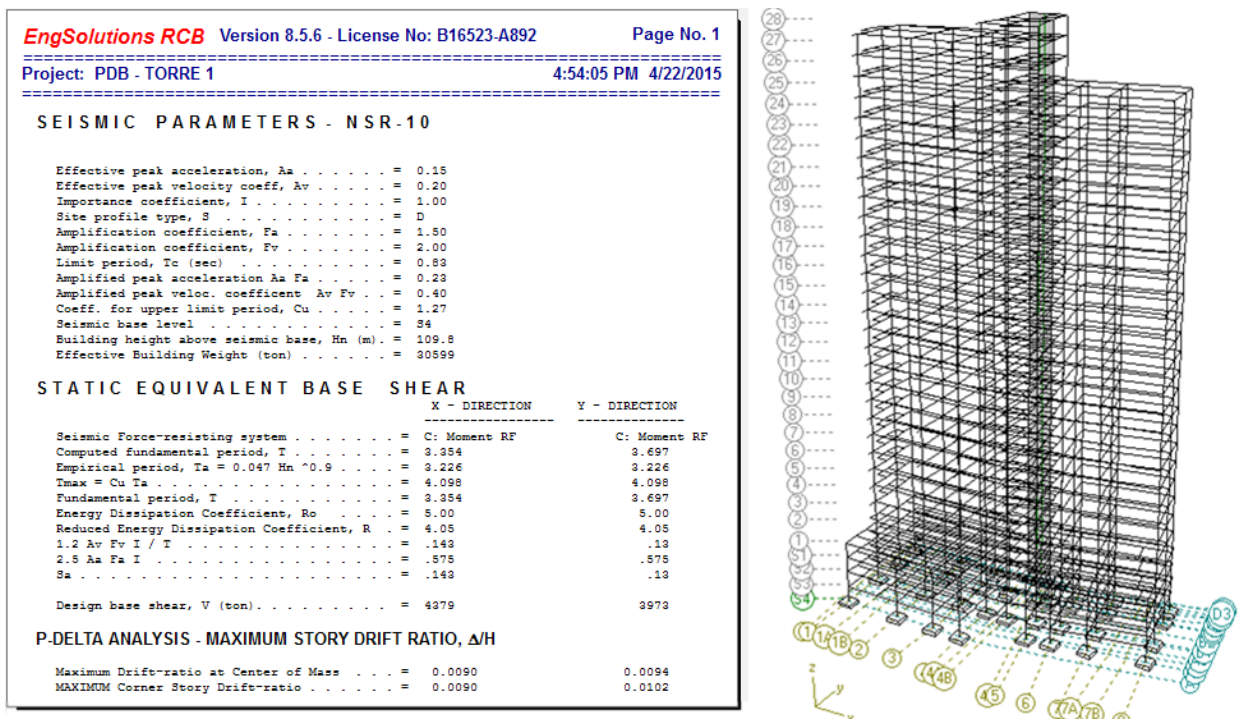

Figura 2. Parámetros sísmicos, análisis fuerza horizontal equivalente y modelo matemático de estructura ejemplo Fuente: elaboración propia

En la figura 3 se presentan, para cada piso de la estructura, los valores de aceleraciones calculadas según la NSR-10 [4], ASCE 7-10 [5], UBC-97 [6], el Eurocódigo 
8-04 [7] y NZS1170.5-04 [8]. Cuando se comparan estos valores, en la figura se observa que la diferencia con respecto a la NSR-10 es significativa. Las aceleraciones de piso calculadas según las demás normas sísmicas llegan a ser más de cuatro veces mayores a las correspondientes a las de la norma NSR-10. Por otra parte, la variación de las aceleraciones con la altura en la NSR-10 es completamente diferente a la correspondiente a las demás normas. Para el ejemplo considerado, las aceleraciones de piso según NSR-10 disminuyen con la altura hasta $h_{x} / h_{e q} \leq 1,0$, mientras que en las demás normas las aceleraciones de piso siempre aumentan con la altura.

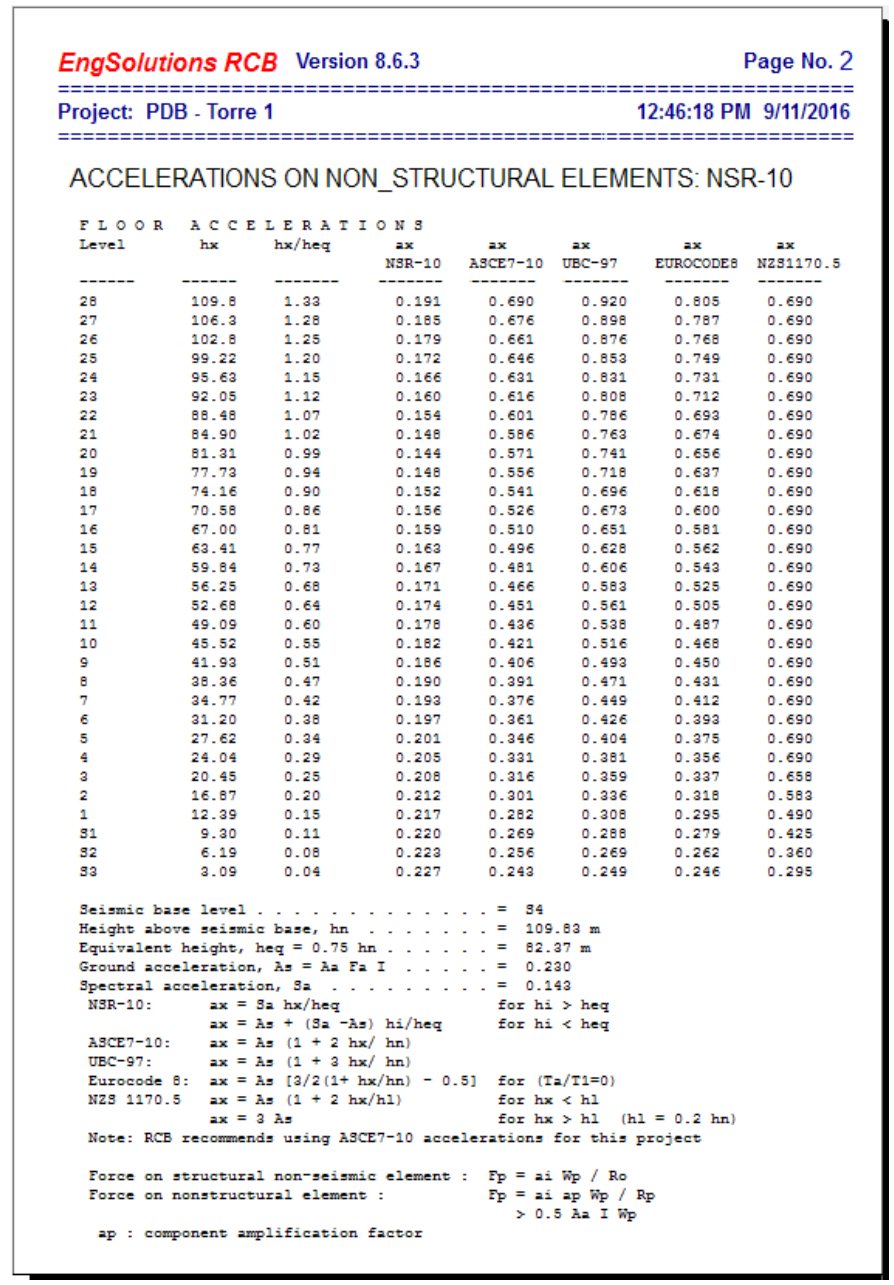

Figura 3. Aceleraciones de piso para el diseño de elementos no estructurales según NSR-10 y otras normas sísmicas

Fuente: elaboración propia 


\subsection{Caso de estudio 2: comparación entre predicciones de reglamentos y aceleraciones me- didas en ensayos en mesa vibratoria}

Entre los años 1970 y 1990 se llevaron a cabo una serie de experimentos en el simulador sísmico de la Universidad de Illinois (ver tabla 1). Este simulador se diseñó para someter modelos de estructuras a escala reducida a aceleraciones horizontales en la base [13]. En los ensayos con este simulador se han obtenido resultados de la respuesta de diferentes modelos de estructuras de concreto reforzado de varios pisos, correspondientes a diferentes configuraciones y diversos sistemas de resistencia lateral. Los resultados obtenidos en estas pruebas han sido usados para proponer valores máximos de aceleraciones de piso, necesarias para diseño de elementos no estructurales [14]. En cada una de las pruebas indicadas en la tabla 1, se ensayaron dos o más pórticos planos conectados a la plataforma de la mesa vibratoria para representar una condición de base fija. El refuerzo longitudinal y transversal de vigas y columnas consistió en alambre de acero con cuantías dentro del intervalo usual en edificaciones reales. El refuerzo longitudinal se hizo continuo en los nudos y se extendió más allá de los nudos exteriores en 'talones' para proveer anclaje. En todos los elementos se colocó suficiente refuerzo transversal para evitar fallas por cortante antes de falla por flexión. Cada estructura fue sometida a múltiples simulaciones (corridas) que consistieron en aumentar la intensidad en corridas sucesivas. Los movimientos aplicados en la base fueron seleccionados de registros de sismos reales. Los resultados de las historias de aceleración y desplazamiento medidas en cada piso para cada ensayo se presentan en forma detallada en las referencias de la tabla 1 [25]. Adicionalmente, los resultados se presentan en forma resumida por Lepage et al. [14].

Tabla 1. Descripción de estructuras ensayadas

\begin{tabular}{|l|l|l|}
\hline \multicolumn{1}{|c|}{ Tipo de sistema } & \multicolumn{1}{|c|}{ Estructuras } & \multicolumn{1}{c|}{ Fuente } \\
\hline \multirow{3}{*}{ Muros acoplados } & D1, D2, D3, M1 & Aristizábal y Sozen [16] \\
\cline { 2 - 3 } & D1, D2, D3, D4, D5 & Lybas y Sozen [17] \\
\hline \multirow{4}{*}{ Combinado: pórticos-muros } & FW1, FW2, FW3, FW4 & Abrams y Sozen [18] \\
\cline { 2 - 3 } & FSW, FHW, FFW & Moehle y Sozen [19] \\
\hline \multirow{4}{*}{ Pórticos con vigas fluyendo } & MF1 & Healey y Sozen [20] \\
\cline { 2 - 3 } & MF2 & Moehle y Sozen [21] \\
\cline { 2 - 3 } & H1, H2 & Cecen [22] \\
\cline { 2 - 3 } & FNW & Mohele y Sozen [18] \\
\hline & SS1, SS2 & Schultz [22] \\
\cline { 2 - 3 } & Tower, Stepped & Wood [23] \\
\cline { 2 - 3 } & ES1, ES2 & Eberhard y Sozen [24] \\
\hline
\end{tabular}

Fuente: elaboración propia 
En la figura 4 se presentan los valores de aceleración pico en cada piso para cada una de las simulaciones. Las aceleraciones (eje horizontal) fueron normalizadas con respecto a la aceleración pico del terreno. La elevación del piso (eje vertical) se normalizó con respecto a la altura total de la estructura (altura del techo). El eje horizontal en la figura 4 representa la amplificación de la aceleración de cada piso con respecto a la aceleración en la base. En la figura se observa que, en todos los casos, las máximas amplificaciones de la aceleración ocurren en los pisos superiores.

En la figura 5 se comparan los resultados de aceleraciones máximas de piso medidas en todas las pruebas, con la predicción de las diferentes normas analizadas en este estudio. Esta figura muestra, por una parte, que las ecuaciones para determinar las aceleraciones de piso para el diseño de elementos no estructurales de las normas ASCE 7-10 [5], UBC-97 [6], el Eurocódigo 8-04 [7] y NZS 1170.5-04 [8] son representativas de la envolvente de las aceleraciones de piso. Por otra parte, en la misma figura se observa que las aceleraciones de piso calculadas según la noma NSR-10 [4], para el ejemplo considerado, son significativamente menores que la gran mayoría de los valores experimentales.
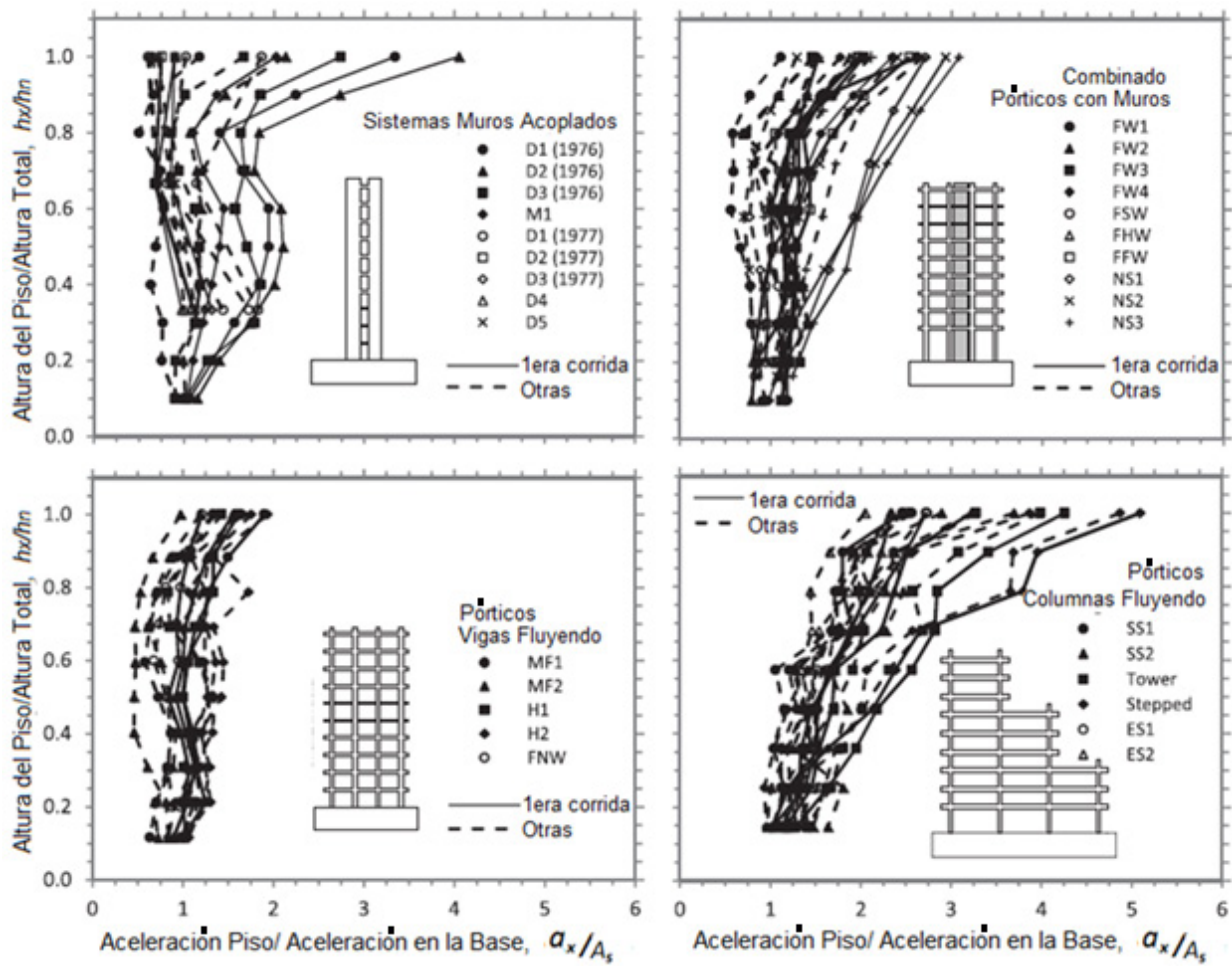

Figura 4. Aceleraciones máximas en los pisos medidas en las pruebas para cada tipo de estructura Fuente: elaboración propia 


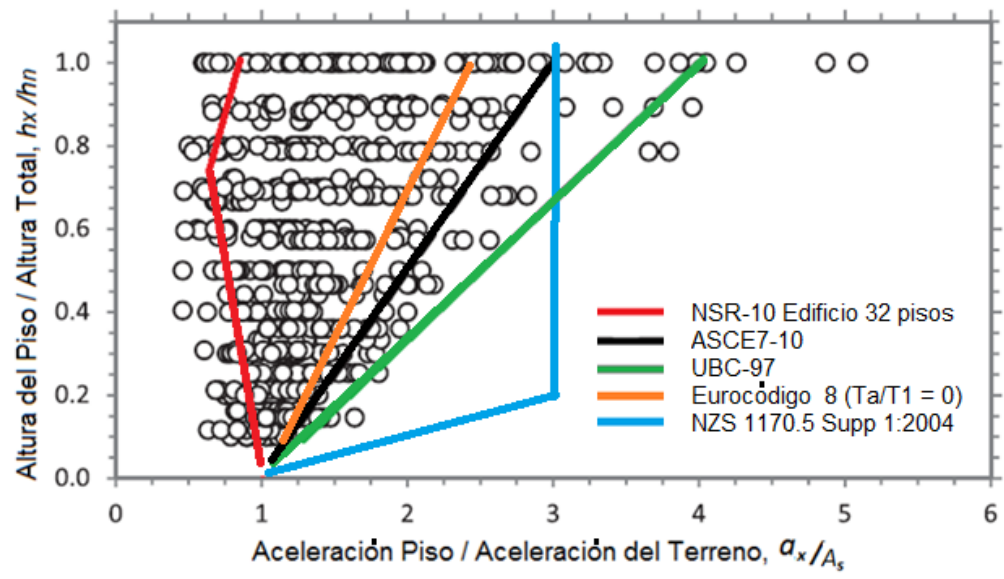

Figura 5. Valores experimentales de aceleración máxima del piso comparada con fórmulas en normas

Fuente: elaboración propia

\subsection{Caso de estudio 3: comparación entre predicciones de reglamentos y aceleraciones medidas en edificaciones reales de 5 a 20 pisos}

Lepage et al. [14] han reportado las aceleraciones de piso medidas en siete edificaciones de concreto reforzado localizadas en el área de Los Ángeles, California, durante sismos reales. Las características de las edificaciones se presentan en la tabla 2. Estas mediciones se encuentran disponibles en la página web del CESMD [26]. En este estudio se han comparado estas aceleraciones con los valores predichos por las fórmulas en las normas analizadas. Los datos corresponden a aceleraciones medidas durante cuatros sismos: San Fernando de 1971, Whittier de 1987, Sierra Madrid de 1991 y Northridge de 1994. En esta comparación solo se consideraron los resultados de los registros en los que la aceleración del terreno excedió $0,1 \mathrm{~g}$.

Para robustecer la base de datos, en este estudio también se usaron los valores medidos de las amplificaciones de aceleración compiladas por Drake y Backman $[27,28]$, las cuales se utilizaron para la elaboración de las provisiones NEHRP. Estas provisiones se basan en 405 juegos de registros de aceleraciones de piso obtenidas en diferentes edificaciones instrumentadas durante 16 sismos fuertes en California. Con un formato similar a la figura 5, en la figura 6 se presentan todas las aceleraciones de piso máximas medidas. Las aceleraciones de piso en la figura están normalizadas con respecto a la aceleración máxima en la base (eje X) y la altura del piso está normalizada con respecto a la altura del techo. De modo similar a los resultados medidos en los ensayos en mesa vibratoria de las estructuras (figura 5), en la figura 6 se observa que las máximas amplificaciones ocurren siempre en el techo, excepto en NSR-10. 
Tabla 2. Descripción de edificios existentes donde se midieron las aceleraciones

\begin{tabular}{|l|c|c|c|c|}
\hline \multicolumn{1}{|c|}{ Edificio } & $\begin{array}{c}\text { Identificación } \\
\text { CESMD }\end{array}$ & $\begin{array}{c}\text { Sistema de resistencia } \\
\text { sísmica }\end{array}$ & Altura en $m$ & Pisos \\
\hline Bodega Los Ángeles & 24463 & PRM & 36 & 5 \\
\hline Parqueadero Los Ángeles & 24655 & ME & 19 & 6 \\
\hline Hotel Van Nuys & 24386 & PRM & 20 & 7 \\
\hline Residencial Burbank & 24385 & ME & 27 & 10 \\
\hline Sherman Oaks & 24322 & PRM & 50 & 13 \\
\hline Almacén Hollywood & 24236 & PRM & 42 & 14 \\
\hline Hotel North Hollywood & 24464 & PRM & 59 & 20 \\
\hline
\end{tabular}

$\mathrm{PRM}=$ pórticos resistentes a momento, $\mathrm{ME}=$ muros estructurales

Fuente: elaboración propia

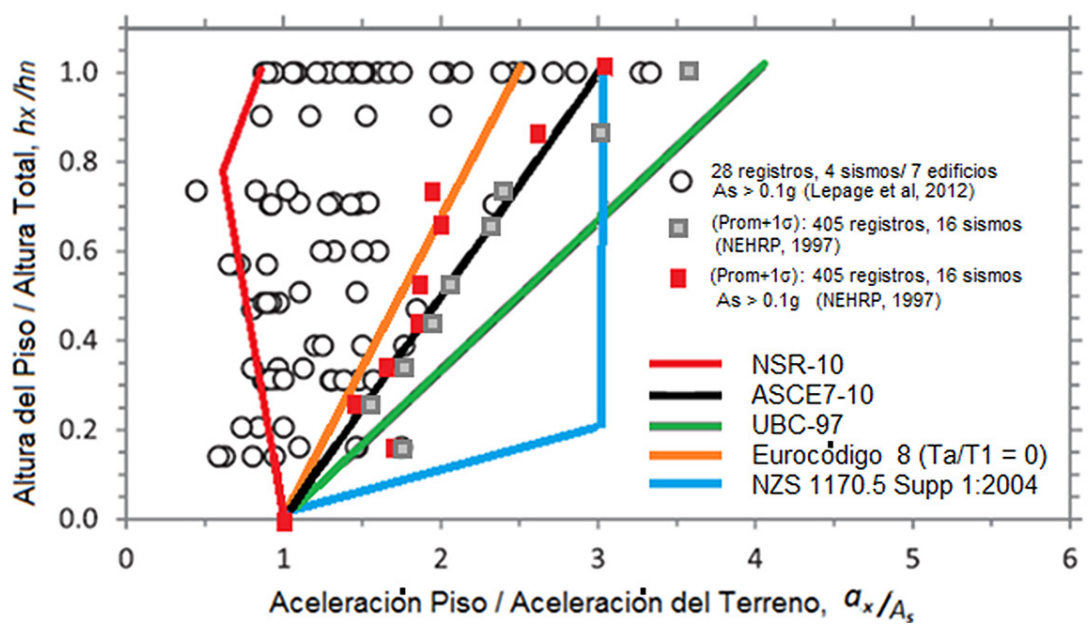

Figura 6. Aceleraciones de piso medidas en edificaciones existentes comparadas con fórmulas en normas

Fuente: elaboración propia

Para esta base de datos sísmicos se obtuvo la distribución con la altura del valor promedio más desviación estándar $(\operatorname{Prom}+1 \sigma)$ de la relación entre la aceleración máxima de cada piso y la aceleración máxima en la base $\left(a_{x} / A_{s}\right)$. En la figura 6 se muestra la distribución original de los valores del Prom $+1 \sigma$ que sirvió de base para las provisiones en el UBC-97, la cual incluye la totalidad de los registros. En la figura se incluye la distribución modificada de los valores del Prom $+1 \sigma$ que sirvió de base para ASCE 7-10, en la cual solo se consideraron registros correspondientes a aceleraciones máximas en la base superiores a 0,1 g. En la figura 6 también se muestran los resultados 
obtenidos con las ecuaciones de las normas ASCE 7-10, el Eurocódigo 8-04, UBC-97 y NZS 1170.5-04. Se observa que los resultados de las aceleraciones máximas de piso obtenidas con estas ecuaciones son representativas de la envolvente de aceleraciones medidas. Adicionalmente, en la figura se observa que las predicciones de las ecuaciones de UBC-97 y NZS 1170.5-04 son las más conservadoras. Por otra parte, en la figura se aprecia que, para el ejemplo considerado, las aceleraciones calculadas según la noma NSR-10 nuevamente son significativamente menores que los valores medidos. De hecho, las aceleraciones en el techo calculadas según la NSR-10 son menores que la totalidad de las aceleraciones medidas en edificaciones reales.

\subsection{Caso de estudio 4: comparación entre predicciones de reglamentos y aceleraciones medidas en una edificación real de 20 pisos}

En el cuarto caso considerado en este estudio se comparan las aceleraciones de piso calculadas con las diferentes normas analizadas, con las aceleraciones reales medidas en un edificio de mediana altura. En este estudio se seleccionó el caso de un hotel de 20 pisos localizado en el norte de Hollywood. Como se observa en la figura 7, el sistema de resistencia sísmica del edificio corresponde a pórticos dúctiles de concreto reforzado. La estructura es representativa de un gran número de edificaciones existentes en Colombia. Se trata de una estructura relativamente flexible que, aunque cumple con los límites de deriva del ASCE 7-10, no cumple con los límites de deriva de la NSR-10. De acuerdo con Goel y Chopra [29], los periodos de vibración reales medidos durante el sismo de Northridge de 1994, son $T_{x}=2.60 \mathrm{~s}$ (longitudinal, N-S) y $T_{y}=2.62 \mathrm{seg}$ (transversal, E-W).

CGS CSMIP-24464

North Hollywood - 20-story Hotel

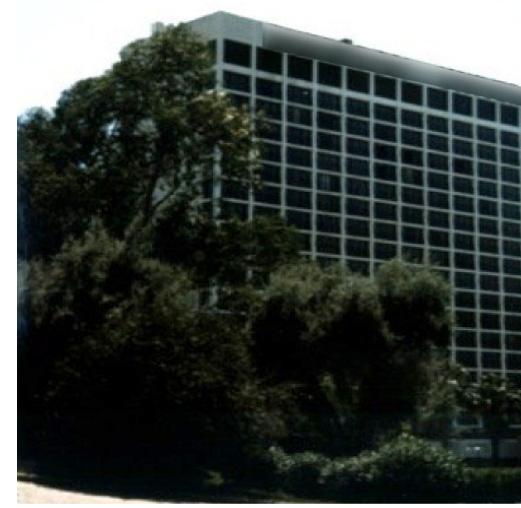

EngSalutions ACB 8.6 .3

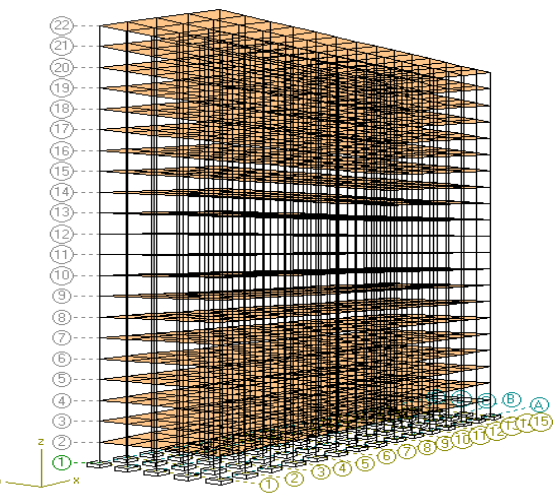

Figura 7. Hotel en North Hollywood: (a) fotografía [26], (b) modelo estructural en RCB V8.5 Fuente: elaboración propia 
Los registros de aceleraciones de piso obtenidos en este edificio durante cuatros sismos fuertes en California están disponibles en la página web del CESMD [26]. El número de identificación en el CESMD del edificio aquí analizado es CSMIP-24464. En este estudio, la comparación se hace para las aceleraciones medidas durante el sismo de Northridge 1994, en el cual se registró daño extensivo en las componentes no estructurales de este edificio. En la figura 8 se presentan los registros de aceleración de piso medidos durante el sismo de Northridge de 1994. En la figura se observa claramente que, en las dos direcciones, la aceleración en el techo excede ampliamente la aceleración en el piso.

Las aceleraciones máximas del terreno $\left(A_{x}\right)$ para el sitio fueron $0,32 \mathrm{~g}$ en la dirección norte-sur (N-S) y $0,11 \mathrm{~g}$ en la dirección este-oeste (E-W). En este caso, el hotel está localizado en un perfil de suelo tipo C. Según la NSR-10, para Bogotá y Medellín, las aceleraciones máximas del terreno $\left(A_{a} \times F_{a}\right)$ para los perfiles de suelo serían: perfil $\mathrm{A}=$ $0,12 \mathrm{~g}(0,15 \times 0,8)$, perfil $\mathrm{B}=0,15 \mathrm{~g}(0,15 \times 1,0)$, perfil $\mathrm{C}=0,18 \mathrm{~g}(0,15 \times 1,2)$, perfil $\mathrm{D}=0,22 \mathrm{~g}$ $(0,15 \times 1,5)$ y perfil $\mathrm{E}=0,32 \mathrm{~g}(0,15 \times 2,1)$. Por tanto, el intervalo de aceleraciones máximas del terreno registradas en el edificio de estudio $(0,11 \mathrm{~g}$ y $0,32 \mathrm{~g})$, es representativo de las aceleraciones $A_{x}$ que se esperan para el sismo de diseño en las dos ciudades más densamente pobladas de Colombia: Bogotá y Medellín.

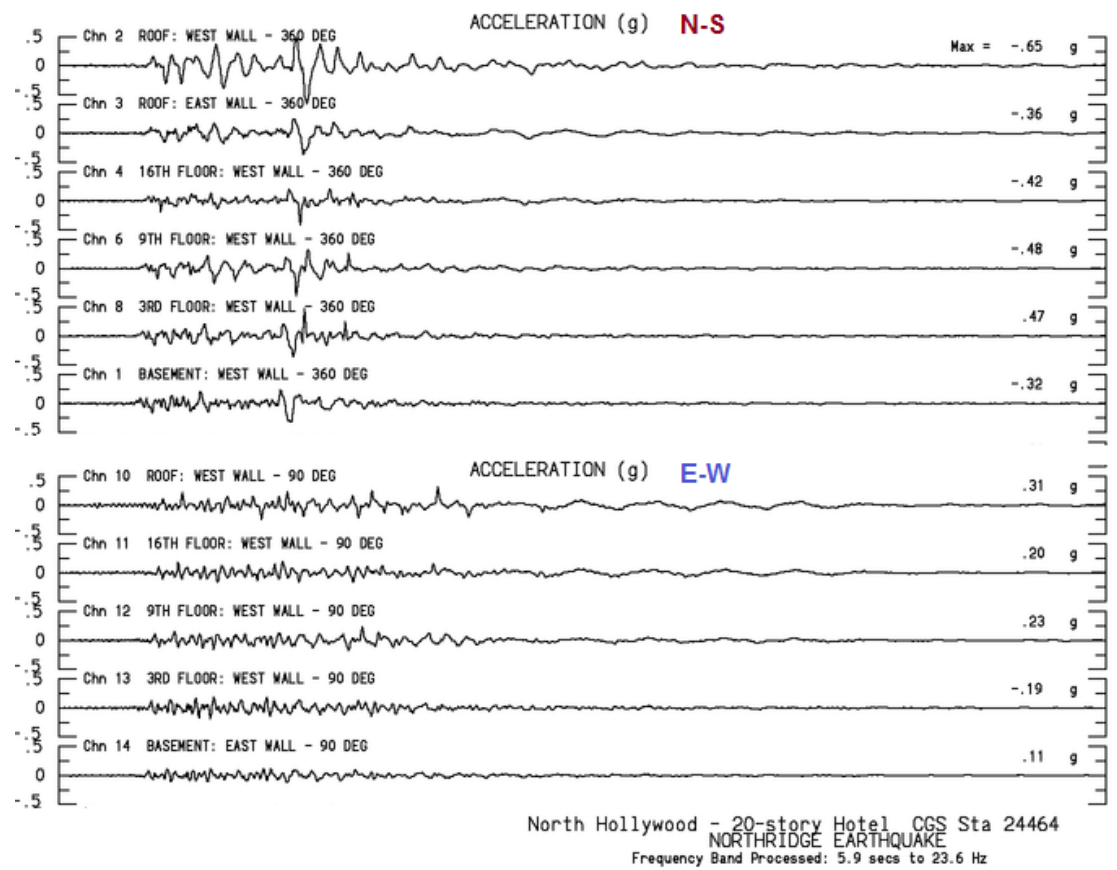

Figura 8. Registros de aceleraciones de piso para sismo de Northridge de 1994 
En la figura 9 se presentan, para las dos componentes (E-W, y N-S), las máximas aceleraciones de piso registradas en varios pisos, normalizadas con respecto a la aceleración del terreno $\left(A_{x} / A_{s}\right)$. En esta figura también se presenta la relación de aceleraciones calculadas según la norma NSR-10 para los parámetros sísmicos representativos del movimiento sísmico que experimentó la estructura; es decir, $A_{a}=0.29 \mathrm{~g}$ y Perfil C, lo cual resulta en una aceleración del terreno $A_{s}=0.32 \mathrm{~g}\left(A_{a} \times F_{a}\right)$. En la figura 9 también se presenta la relación de aceleraciones calculada según la NSR-10 para zonas de amenaza sísmica intermedia ( $A_{a}=0,15 \mathrm{~g}$ ) en perfiles $\mathrm{A}$ (roca) y $\mathrm{E}$ (suelo blando), representativas del intervalo de aceleraciones de diseño de Bogotá y Medellín. Por otra parte, en esta figura se presenta la distribución de aceleraciones según ASCE 7-10, UBC-97, Eurocódigo 8-04 y la norma neozelandesa NZS 1170.5-04.

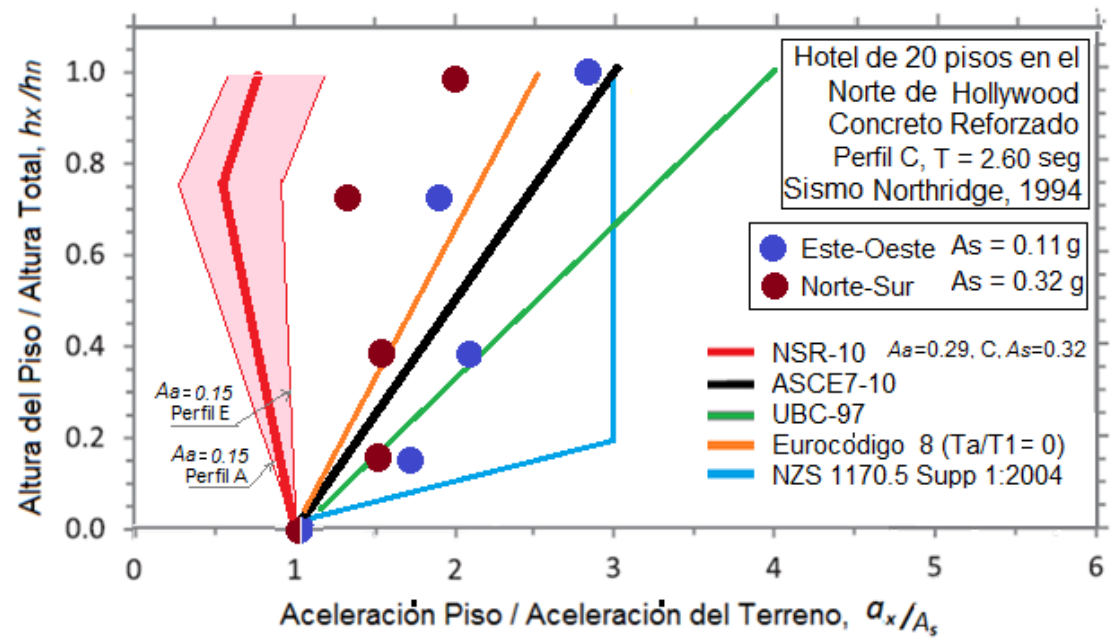

Figura 9. Comparación entre aceleraciones de piso medidas en hotel de 20 pisos en el norte de Hollywood y las fórmulas de reglamentos

Fuente: elaboración propia

En la figura 9 se observa que las aceleraciones registradas en el edifico estudiado son significativamente mayores que las aceleraciones predichas por la NSR-10. En la figura se observa que todos los valores de aceleración registrados están totalmente fuera y lejos del intervalo de aceleraciones calculado con la NSR-10 para perfiles de suelo A hasta E. Por el contrario, las ecuaciones de las normas ASCE 7-10, UBC-97, el Eurocódigo 8-04 y NZS 1170.5-04 son, en general, representativas de la envolvente de las aceleraciones medidas. En la figura se observa que, para bajas relaciones de altura $h_{x} / h_{n}$, las aceleraciones medidas superan los valores obtenidos según estas normas, sin embargo, el valor mínimo de la fuerza inercial $F_{p}$ especificado en las normas cubre esta deficiencia. 


\section{CONCLUSIONES Y RECOMENDACIONES}

Los resultados de este estudio demuestran que las ecuaciones indicadas en la NSR-10 para calcular las aceleraciones de piso que son necesarias para diseñar elementos no estructurales en edificios altos y de mediana altura son significativamente menores que las aceleraciones requeridas en otras normas, incluyendo la norma americana vigente ASCE 7-10, el UBC-97, el Eurocódigo 8-04 y la norma neozelandesa NZS 1170.5-04.

Por otra parte, las aceleraciones de piso calculadas según la norma NSR-10 son significativamente menores que las aceleraciones máximas de piso medidas experimentalmente en pruebas en mesa vibratoria, y que las aceleraciones máximas de piso medidas en edificaciones existentes durante sismos reales. Para edificaciones de mediana altura, la aceleración de piso calculada según la norma NSR-10 es menor que todas las aceleraciones medidas en edificaciones reales consideradas en este estudio. Por el contrario, las aceleraciones de piso calculadas según las normas ASCE 7-10, UBC-97, el Eurocódigo 8-04 y NZS 1170.5-04 son representativas de la envolvente de las aceleraciones medidas experimentalmente y registradas en edificaciones reales.

Los resultados de este estudio también demuestran que la variación de las aceleraciones de piso con la altura que fue obtenida con las fórmulas de la NSR-10, se aleja significativamente de las distribuciones consideradas en otras normas, de las distribuciones reales medidas experimentalmente en pruebas en mesa vibratoria y de las observadas en estructuras existentes. En las normas estudiadas se observó consistencia entre los resultados experimentales y mediciones en edificaciones existentes ya que, independientemente del número de pisos, la aceleración máxima de piso siempre se amplifica con la altura. Sin embargo, en la norma NSR-10, la distribución está determinada por la magnitud de la aceleración espectral $S_{a} \mathrm{y}$, por tanto, esta distribución depende del periodo fundamental y de la altura del edificio. Para edificios de mediana altura y edificios altos en los que la ordenada espectral está en la rama descendiente del espectro de diseño, como en el caso del ejemplo considerado, las fórmulas de la NSR-10 resultan en una reducción de la aceleración con la altura del piso. Se debe anotar que los desplazamientos laterales máximos y las fuerzas internas máximas del sistema estructural están controlados por los primeros modos de vibración del sistema de resistencia sísmica integrado. Sin embargo, los efectos inerciales de las componentes no estructurales están controlados por las aceleraciones máximas de los pisos individuales, las cuales están controladas por los modos de vibración superiores, cuyos periodos corresponden a la meseta del espectro.

Es importante también anotar que, para edificios medianamente altos como los analizados en las figuras 5, 6 y 9, el margen de error en la magnitud de las aceleraciones 
de piso calculadas con NSR-10 puede ser significativo. En casos de edificios más altos y en suelos blandos, el error es aún mayor que el mostrado en este estudio. Esto impide un diseño seguro de los elementos no estructurales, pues se subestima drásticamente el efecto inercial del sismo, que es la principal causa de la falla y caída de estos elementos. Si se considera el alto riesgo asociado a la falla de estos elementos ante un sismo fuerte, incluyendo riesgo a la vida, el riesgo de pérdidas económicas importantes por daño a los edificios y su contenido, y el riesgo de pérdida temporal de funcionalidad, se hace prioritario incluir rápidamente modificaciones a la norma NSR-10. De esta manera, en este artículo se recomienda incluir en la NSR-10 las siguientes modificaciones:

- Modificar la ecuación A.9.4-2 para calcular las aceleraciones para diseño de elementos no estructurales, de tal manera de adoptar la distribución usada actualmente en ASCE 7-10; es decir:

$$
\begin{gathered}
a_{x}=A_{s}\left(1+2 \frac{h_{x}}{h_{n}}\right) \\
\mathrm{A}_{\mathrm{s}}=\mathrm{A}_{\mathrm{a}} \mathrm{F}_{\mathrm{a}} \mathrm{I}
\end{gathered}
$$

- Modificar la sección A.9.4.8, de tal manera que los elementos no estructurales localizados por debajo de la base sísmica se diseñen para una aceleración $a_{x}$ igual a $A_{s}=A_{a} F_{a} I$.

- Modificar la ecuación A.9.4-1 para que el valor mínimo de la fuerza horizontal sea $F_{p}=A_{s} g M_{p}$.

- Modificar las ecuaciones A.8.2-1 y A.3.6-3 para adoptar la misma ecuación indicada en (a).

- Especificar que se debe incluir en los planos estructurarles las aceleraciones $a_{x}$ que se deben usar para diseñar los soportes de los componentes mecánicos, eléctricos, hidráulicos y arquitectónicos.

\section{AGRADECIMIENTOS}

Los autores agradecen a los ingenieros Luis E. Huertas, Carlos E. Blodek y Adriana C. Hoyos por sus observaciones, comentarios, sugerencias y aportes en la preparación del artículo. El tercer autor agradece a la Vicerrectoría de Investigaciones de la Universidad Militar Nueva Granada por el apoyo en las actividades de investigación. 


\section{REFERENCIAS}

[1] M. Rodríguez, J. Restrepo y J. Carr, "Earthquake-induced floor horizontal accelerations in buildings," Journal of Earthquake Engineering and Structural Dynamics, vol. 31, pp. 693-718, 2002.

[2] J. Jaramillo, "Evaluación aproximada de la aceleración absoluta en sistema de múltiples grados de libertad considerando la participación de n formas modales," Revista Internacional de Desastres Naturales, Accidentes e Infraestructura Civil, vol. 4, №. 2, pp. 87-98, 2005.

[3] J. Carrillo y G. González, "Evaluación de la demanda sísmica fuera del plano en edificios," Revista Ciencia e Ingeniería Neogranadina, vol. 15, pp. 44-61, 2005.

[4] NSR-10, Reglamento Colombiano de Construcción Sismo Resistente-NSR-10, Asociación Colombiana de Ingeniería Sísmica, AIS, Tomo 1, Bogotá, 2010.

[5] ASCE 7-10, Minimum design loads for buildings and other structures, ASCE/SEI 7-10, Reston, VA, 2010.

[6] UBC-97, "Uniform building code - Vol. 2: Structural engineering design provisions," presentado en International Conference of Building Officials, Whittier, California, EE. UU., 1997.

[7] Eurocódigo 8-04, Design of structures for earthquake resistance, European Committee for Standardization, 2004.

[8] NZS 1170.5-04, New Zealand Standard: Structural design actions - Part 5: Earthquake actions. Standards, Nueva Zelanda, 2004.

[9] FEMA, Reducing de risks of nonstructural earthquake damage -A practical guide, FEME E-74, Washington, 2012.

[10] FEMA, NEHRP recommended provisions for seismic regulations for new buildings and other structures, Part 1: Provisions, FEMA 302, Washington, 1997.

[11] FEMA, NEHRP recommended provisions for seismic regulations for new buildings and other structures, Part 2: Commentary, FEMA 303, Washington, 1997.

[12] EngSolutions RCB, Structural software for analysis and design of reinforced concrete buildings for earthquake and wind forces, V8.5, EngSolutions, Inc., Florida, 2015.

[13] M. Sozen, S. Otani, P. Gulkan y N. Nielsen, "The University of Illinois earthquake simulator," presentado en Proceedings of the 4th World Conference on Earthquake Engineering, Santiago de Chile, vol. III, 1969.

[14] A. Lepage, J. Shoemaker y A. Memari, “Accelerations of nonstructural components during nonlinear seismic response of multistory structures," Journal of Architectural Engineering, ASCE, vol. 18, №. 4, pp. 285-297, 2012.

[15] J. Aristizabal y M. Sozen, "Behavior of ten-story reinforced concrete walls subjected to earthquake motions", Structural Research Series, N. 431, Univ. of Illinois, Urbana, Illinois, 1976. 
[16] J. Lybas y M. Sozen, "Effect of beam strength and stiffness on dynamic behavior of reinforced concrete coupled walls," Structural Research Series, №. 444, Univ. of Illinois, Urbana, Illinois, 1977.

[17] D. Abrams y M. Sozen, "Experimental study of frame-wall interaction in reinforced concrete structures subjected to strong earthquake motions," Structural Research Series, n. 460, Univ. of Illinois, Urbana, Illinois, 1979.

[18] J. Moehle y M. Sozen, "Experiments to study earthquake response of R/C structures with stiffness interruptions," Structural Research Series, n. 482, Univ. of Illinois, Urbana, Illinois, 1980.

[19] T. Healey y M. Sozen, "Experimental study of the dynamic response of a ten-story reinforced concrete frame with a tall first story," Structural Research Series, n. 450, Univ. of Illinois, Urbana, Illinois, 1978.

[20] J. Moehle y M. Sozen, "Earthquake simulations tests of a ten-story reinforced concrete frame with discontinued first-level beam," Structural Research Series, n. 451, Univ. of Illinois, Urbana, Illinois, 1978.

[21] H. Ceceen, "Response of ten-story reinforced concrete model frames to simulated earthquakes," Ph.D. thesis, Univ. of Illinois, Urbana, Illinois, 1979.

[22] A. Schultz, "An experimental and analytical study of the earthquake response of R/C frames with yielding columns," Ph.D. thesis, Univ. of Illinois, Urbana, Illinois, 1985.

[23] S. Wood, "Experiments to study the earthquake response of concrete frames with setbacks," Ph.D. thesis, Univ. of Illinois, Urbana, Illinois, 1986.

[24] M. Eberhard y M. Sozen, "Experiments and analyses to study the seismic response of reinforced concrete frame-wall structures with yielding columns," Structural Research Series No. 548, Univ. of Illinois, Urbana, Illinois, 1989.

[25] NEEShub, "The George E. Brown, Jr. Network for Earthquake Engineering Simulation," [En línea], Disponible: https://nees.org/warehouse, 2017.

[26] CESMD, “Center for engineering strong motion data,”USGS, CGS, ANSS. [En línea], Disponible: https://www.strongmotioncenter.org, 2017.

[27] R. Drake y R. Bachman, "1994 NEHRP Provisions for Architectural, Mechanical, and Electrical Components," presentado en Proceedings of the $5^{\text {th }}$ U.S. National Conference on Earthquake Engineering, 1994.

[28] R. Drake y R. Bachman, "Interpretations of instrumented building seismic data and implications for building codes," presentado en Proceedings of the 1995 SEAOC Annual Convention, 1995.

[29] R. Goel y A. Chopra, "Period Formulas for Moment-Resisting Frame Buildings," Journal Structural Engineering, ASCE, vol. 123, N. 11, pp. 1454-1461, 1997. 